\title{
Diurnal variation in the pharmacokinetics and brain distribution of morphine and its major metabolite
}

Laura Kervezee ${ }^{1,2}$; Robin Hartman²; Dirk-Jan van den Berg ${ }^{2}$; Johanna H. Meijer ${ }^{1,3,{ }^{*}}$; Elizabeth C.M. de Lange $\mathrm{e}^{2,3, *}$

${ }^{1}$ Laboratory for Neurophysiology, Department of Molecular Cell Biology, Leiden University Medical Center, Leiden, the Netherlands;

${ }^{3}$ Division of Pharmacology, Leiden Academic Center for Drug Research, Leiden University, Leiden, the Netherlands

${ }^{3}$ These authors share senior authorship

*Corresponding authors, contact details:

- $\quad$ Prof. Dr. J.H. Meijer, Laboratory for Neurophysiology, Department of Molecular Cell Biology, Leiden University Medical Center, PO Box 9600 Mailbox S5-P, 2300 RC, Leiden, The Netherlands, Tel: +31 71526 9760, Mail: J.H.Meijer@lumc.nl

- $\quad$ Dr. E.C.M. de Lange, Division of Pharmacology, LACDR, Leiden University, PO Box 9502, 2300 RA, Leiden, The Netherlands, Tel: +31 715276330 Mail: ecmdelange@lacdr.leidenuniv.nl 


\section{Abstract}

The pharmacokinetics and pharmacodynamics of drugs are influenced by daily fluctuations in physiological processes. The aim of this study was to determine the effect of dosing time on the pharmacokinetics and brain distribution of morphine. To this end, $4 \mathrm{mg} / \mathrm{kg}$ morphine was administered intravenously to Wistar rats that were either pre-treated with vehicle or tariquidar and probenecid to inhibit processes involved in the active transport of morphine. Non-linear mixed effects modelling was used to describe the concentration-time profiles of morphine and its metabolite M3G in plasma and brain tissue. We found that the concentrations of morphine in the brain and of M3G in plasma depended on the time of day, which could be quantified by a 24-hour rhythm in the efflux of morphine from brain tissue back into the circulation, with the lowest efflux during the two light-dark phase transitions with a difference between peak and trough of $20 \%$. The active processes involved in the clearance of morphine and its metabolite M3G from plasma also showed 24-hour variation with the highest value in the middle of the dark phase being $54 \%$ higher than the lowest value at the start of the light phase. Hence, time of day presents a considerable source of variation in the pharmacokinetics of morphine, which could be used to optimize the dosing strategy of morphine.

Keywords: circadian rhythms, personalized medicine, chronopharmacology, morphine, pharmacokinetic modelling 


\section{Introduction}

Morphine is the most widely used opioid for the treatment of moderate to severe pain, despite the many side-effects associated with its use. Establishing a dosing regimen that results in adequate analgesia and minimal adverse side-effects is crucial, but this remains a challenge due to the high degree of intra- and interindividual variability associated with its pharmacokinetics and pharmacodynamics [1]. Time of day presents a considerable source of variation in the pharmacokinetics and pharmacodynamics of a wide variety of drugs due to daily rhythms in physiological processes [2].

There are several indications that time of day influences morphine's effect in both humans and animal models [3-14]. However, the physiological mechanisms that underlie these variations in morphineinduced analgesia are unknown. To gain a more structured overview of the effect of time of drug administration on the therapeutic effect of morphine, it is essential to first determine 24-hour variation in both the plasma and brain pharmacokinetics of morphine. Although the effect of time of administration on the exposure to morphine has previously received some attention $[15,16]$, these studies neither determined the 24-hour variation in the different pharmacokinetic parameters of morphine, nor did they address the 24-hour variation in its distribution to the brain, morphine's main site of action.

The concentration of morphine in blood and subsequently in the central nervous system depends on several processes, such as metabolism by UDP glucuronosyl transferase (UGT) $2 \mathrm{~B} 7$ in the liver $[17,18]$ and efflux transport by specialized transporters including P-glycoprotein and multi-drug resistance proteins (mrps) [1,19-21]. It has been shown that these physiological processes show 24hour variation $[2,22,23]$.

To determine 24-hour variation in the pharmacokinetic parameters of morphine, we used a study design in which morphine is intravenously administered to rats at six time-points during the 24-hour period combined with a pharmacokinetic modelling approach. Results from this study enhance our understanding of the processes that underlie the observed time-of-day dependent analgesic effect of morphine. 


\section{Methods \& Materials}

\subsection{Animals}

Male Wister WU rats (Charles River, the Netherlands) were housed in groups for at least twelve days under standard environmental conditions (humidity $60 \%$, ambient temperature $21^{\circ} \mathrm{C}$ ) with food (Laboratory chow, Hope Farms, Woerden, The Netherlands) and water ad libitum. After surgery, animals were kept individually until the end of the experiment under otherwise similar conditions. The animal procedures were performed in accordance with the Dutch law on animal experimentation and were approved by the Animal Ethics Committee of the Leiden University (protocol number DEC14041).

\subsection{Study design}

Cannulation of the femoral artery and vein was performed as described previously [24]. Anesthesia was induced and maintained by respectively $5 \%$ and $1-2 \%$ isoflurane throughout the surgical procedures. Experiments were conducted seven days after surgery and started at one of six different time points ( $\mathrm{t}=0$ at either Zeitgeber time (ZT) 0,4, 8, 12, 16 or 20, with ZT12 defined as the moment that lights are turned off). Experiments that took place during the dark phase were conducted under dim red light. At $\mathrm{t}=-25 \mathrm{~min}$, tariquidar (15mg/kg; XR9576 from Avant pharmaceuticals, London, UK, in $5 \%$ glucose) or vehicle ( $5 \%$ glucose) was administered for 10 minutes, followed by administration of probenecid (150mg/kg; Sigma-Aldrich, Zwijndrecht, the Netherlands, in $\left.5 \% \mathrm{NaHCO}_{3}\right)$ or vehicle $(5 \%$ $\mathrm{NaHCO}_{3}$ ) for 10 minutes. Half of the animals received a combination of tariquidar and probenecid (inhibitor-treated group); the other half received the two vehicle solutions (vehicle-treated group). At $\mathrm{t}=0$, morphine $\mathrm{HCl}(4 \mathrm{mg} / \mathrm{kg}$ in saline; Pharmachemie BV, Haarlem, the Netherlands) was administered for 10 minutes. The selection of this dose was based on previous research in our laboratory [25]. All drugs were administered intravenously using a syringe pump (Pump 22 Multiple Syringe Pump, Harvard Apparatus, Holliston, MA, USA). Plasma samples (150 $\mu \mathrm{L})$ were collected at $\mathrm{t}=-5,10,20,30$, $45,60,90$ and $120 \mathrm{~min}$ as well as at $\mathrm{t}=180 \mathrm{and} / \mathrm{or} 240 \mathrm{~min}$, depending on when the experiment was terminated and stored at $-20^{\circ} \mathrm{C}$ until further analysis. At either $\mathrm{t}=120,180$ or $240 \mathrm{~min}$, animals were euthanized by an overdose of Nembutal, transcardially perfused and decapitated. Brain tissue was removed, immediately placed on ice and subsequently stored at $-80^{\circ} \mathrm{C}$ until further analysis. 


\subsection{SPE-LC-MS/MS analysis}

Morphine and morphine-3-glucoronide (M3G) were measured in plasma and brain tissue using liquid chromatography tandem mass spectrometry (LC-MS/MS) (Methods S1).

\subsection{Data processing}

Samples below LLOQ $(<1 \%)$ were marked in the data set but were retained, as described previously [26]. Concentrations $(\mathrm{ng} / \mathrm{mL})$ were converted to nanamol/mL using the molecular weight of morphine. $\mathrm{HCl}(321.8 \mathrm{~g} / \mathrm{mol})$ and M3G (free base) $(461.47 \mathrm{~g} / \mathrm{mol})$. Based on protein binding values of morphine in rats that have been reported previously [27-31], an unbound fraction of $70 \%$ was used. The degree of plasma protein binding of M3G is very low (unbound fraction of 93\% [27]) and was not taken into account in further analysis.

\subsection{Population pharmacokinetic model development}

A population pharmacokinetic model was developed to describe the concentration-time profiles of morphine and $M 3 G$ in plasma and in morphine brain tissue using nonlinear mixed effects modelling (NONMEM 7.3 [32]) in combination with Pirana (v2.8.2), PsN (v3.7.6), Xpose (v4) and R (v3.1.2) [33].

To compare the fit of nested models, the likelihood ratio test was used, under the assumption that the difference in -2 log-likelihood is $x^{2}$ distributed with degrees of freedom (d.f.) determined by the number of additional parameters in the more complex model. Hence, a decrease in Objective Function Value (OFV) of at least 3.84 points ( $p$-value $<0.05$ ) with one additional parameter was considered to provide a significantly better fit of the data compared to its parent model. The fit of non-nested models were compared using the Akaike Information Criterion (AIC) [34].The first-order method with conditional estimation and interaction (FOCEI) and the ADVAN6 subroutine were used throughout model development. Model selection was based on OFV, precision and plausibility of parameter estimates, graphical evaluation of the goodness of fit and visual predictive checks (VPC).

Interanimal variability was described using an exponential model. Additive, proportional and combined error models were considered to describe the residual variability [34]. Pre-treatment with probenecid and tariquidar was assumed to inhibit all active transport processes. Therefore, this effect was assessed on clearance parameters as follows: 


$$
P=\theta_{\text {passive }}+\theta_{\text {active }} *(1-T R T)
$$

Equation 1

Where $\theta_{\text {passive }}$ is the passive component of the clearance parameter, $\theta_{\text {active }}$ is the active component of the clearance parameter and TRT is the treatment group ( 0 for vehicle-treated animals; 1 for inhibitortreated animals). The effect of the inhibitors on other (non-clearance) parameters was assessed as follows:

$$
P=\theta * \theta_{I N H}^{T R T}
$$

Equation 2

Where $\theta_{\mathrm{INH}}$ is the fractional change in parameter $\theta$ in the presence of inhibitor treatment.

A sequential approach was used to develop the population PK model. Firstly, different structural models were considered (different number of peripheral compartments, linear or Michaelis-Menten clearance) to describe morphine and M3G concentrations in plasma. The volume of the M3G compartment was set equal to the volume of the central morphine compartment to yield a structurally identifiable model. In the second step, the morphine concentrations in brain tissue were added to the data set and the PK model was extended to describe the concentration profile in brain tissue. Lastly, the effect of time of day on the pharmacokinetic parameters was assessed. As an exploratory approach, the distribution of conditional weighted residuals with interaction (CWRESI) over time was investigated per treatment group. Subsequently, it was investigated whether the model fit could be improved by describing one or more parameters by a sinusoidal function with a principal period of 24hour and one or more harmonic terms (Equation 3).

$$
P=\theta_{\text {Mesor }}+\sum_{n=1}^{N}\left[\theta_{\text {Amplitude, } n} * \cos \left(\frac{2 \pi * n *\left(t-\theta_{\varphi, n}\right)}{24}\right)\right]
$$

In this equation, $\theta_{\text {Mesor }}$ represents the rhythm-adjusted mean value of the parameter, $N$ is the total number of harmonics included in the model, $\theta_{\text {Amplitude, } n}$ is the amplitude of the $n^{\text {th }}$ harmonic, $\theta_{\varphi, n}$ is the acrophase (time of peak in minutes after onset of light period) of the $\mathrm{n}^{\text {th }}$ harmonic and $\mathrm{t}$ is the time with $t=0$ defined as the onset of the light period.

\subsection{Simulations}

Two dosing regimens were simulated using the package deSolve (v1.11) in R: 1) a single 10min. intravenous infusion of $4 \mathrm{mg} / \mathrm{kg}$ morphine to a rat of $250 \mathrm{~g}$ with dosing at $0,4,8,12,16$ and 20 hours 
after onset of the light period and 2) a continuous infusion of $0.5 \mathrm{mg} / \mathrm{kg} / \mathrm{h}$ to a rat of $250 \mathrm{~g}$ for 24 hours starting at 4 and 16 hours after the onset of the light period.

\section{Results}

\subsection{Morphine and M3G pharmacokinetics in plasma}

Data from three animals were missing due to complications during surgery and data from four animals were missing due to difficulties with the cannulas during the experiment, so data from 89 animals (mean weight \pm standard deviation: $269 \pm 29 \mathrm{~g}$ ) were available for pharmacometric analysis. Table 1 shows the number of animals per treatment group. Morphine and M3G concentrations in plasma are shown in upper and middle panels of Figure 1.

The concentration-time profiles of morphine were described by a model consisting of a central compartment, one peripheral compartment and linear clearance from the body. To account for the difference in morphine pharmacokinetics between vehicle-treated animals and inhibitor-treated animals, the clearance of morphine from the body $\left(\mathrm{CL}_{10}\right)$ was split into an active and a passive component as described in Equation 1, resulting in a significant improvement of the model fit $(\triangle \mathrm{OFV}$ $208, p<0.01,1$ d.f.) and reducing the interanimal variability on $\mathrm{CL}_{10}$ from $123 \%$ to $23 \%$.

It was found that the conversion of morphine to M3G showed concentration-dependent saturation that could be described by Michaelis-Menten kinetics ( $\triangle \mathrm{OFV}-402, \mathrm{p}<0.01,1$ d.f.). Subsequent incorporation of interanimal variability and the effect of inhibitor treatment resulted in a model with interanimal variability included on $\mathrm{CL}_{10}$, the clearance of $M 3 G$ from plasma $\left(\mathrm{CL}_{\mathrm{M} 3 \mathrm{G}}\right)$, intercompartmental clearance $\left(Q_{2}\right)$ and the maximum conversion rate of morphine to $M 3 G\left(V_{\text {max }}\right)$.

\subsection{Morphine pharmacokinetics in brain tissue}

Morphine concentrations in brain tissue in vehicle-treated animals and inhibitor-treated animals at each of the six dosing times are shown in the lower panels of Figure 1. The plasma model was extended to describe these concentration-time profiles. The base model, consisting of one brain compartment and inter-compartmental clearance $\left(Q_{B R}\right)$ to describe the transport to and from plasma, described the brain concentrations poorly and showed high residual unexplained variability $(128 \%)$. In 
subsequent modelling steps, it was found that a model that described the brain concentrations as deep brain concentrations that was indirectly linked to the central plasma compartment by a transit compartment referred to as the extra-cellular fluid (ECF) compartment, known as an important compartment for morphine distribution into the brain [35], could best describe the brain concentrations data. In the final brain model, drug transport between the deep brain compartment and the ECF compartment were described by a single clearance parameter and the flow between the ECF compartment and the plasma compartment by an influx parameter ( $\left.Q_{\mathrm{PL}-E C F}\right)$ and an efflux parameter that was split into a passive ( $\left.Q_{E C F-P L, p a s s i v e}\right)$ and an active $\left(Q_{E C F-P L, a c t i v e}\right)$ component (Figure 2$)$.

The volumes of the ECF and deep brain compartment were fixed to 1, because these values could not be estimated with sufficient precision. This model described the central trend and the variability in the brain concentrations well (Figure 3) and the residual unexplained variability reduced to $13 \%$.

\subsection{Twenty-four hour variation in morphine pharmacokinetics}

The distribution of CWRESI of the morphine concentrations in brain of both vehicle and inhibitortreated animals showed clear time-of-day dependent bias with peaks around the light-dark transitions (Figure 4A). Inclusion of a two-harmonic cosine function with a 24-hour and 12-hour component on the efflux of morphine from the ECF compartment to plasma $\left(Q_{E C F-P L}\right)$ significantly improved the fit of the model ( $\triangle \mathrm{OFV}-16, \mathrm{p}<0.005,4$ d.f.). This cosine function adequately removed this bias (Figure 4B), provided a better fit compared to implementation of a two-harmonic cosine function on the influx parameter $\left(\mathrm{Q}_{\mathrm{PL}-\mathrm{ECF}}\right)(\mathrm{AIC}=-2.7)$ and reduced the residual unexplained variability from $13.1 \%$ to $11.9 \%$. The 24-hour and 12-hour components of this cosine function had a peak at 22.8 hours and 5.9 hours after lights on and relative amplitudes of $4.1 \%$ and $6.3 \%$, respectively (Figure $5 \mathrm{~A}$ ).

The CWRESI of morphine and M3G in plasma did not reveal a time-of-day dependent bias (Figure 4C and E). Nevertheless, inclusion of the same two-harmonic cosine with a 24-hour and 12-hour component on $\mathrm{CL}_{\mathrm{M} 3 \mathrm{G}, \text { active }}$ and $\mathrm{CL}_{10 \text {,active }}$ significantly improved the fit of the model $(\Delta \mathrm{OFV}-28, \mathrm{p}<0.005$, 4 d.f.) and minimally affected the distribution of CWRESI over time-of-day (Figure 4D and F). The 24hour and 12-hour components of this cosine function had a peak at 18 hours and 7.6 hours after lights on and relative amplitudes of $13 \%$ and $12.5 \%$, respectively (Figure 5B and $\mathrm{C}$ ). 
In the final model (Run8202), the cosine function on $Q_{E C F-P L}$ was combined with the cosine functions

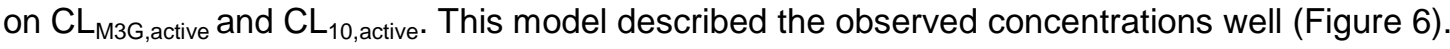
Parameter estimates from this model and from a bootstrap (500 runs) are shown in Table 2.

\subsection{Simulations}

The final model was used to perform simulations to show the impact of dosing time on the concentration profiles of morphine and M3G. Morphine concentrations in plasma after a single intravenous infusion are minimally affected by dosing time, while, morphine concentrations in brain tissue are influenced by dosing time with the highest concentrations attained 12 hours after the onset of the light period (Figure 7A). M3G concentrations were lowest and highest after administration at 4 and 20 hours after the onset of the light period, respectively. During a continuous infusion at steadystate, simulations indicate that morphine and M3G concentrations in plasma and morphine concentrations in brain fluctuate during the 24-hour period (Figure 7B).

\section{Discussion}

In this study, we have been able to characterize the effect of dosing time on processes that are involved in the distribution, metabolism and excretion of morphine through the development of a population pharmacokinetic model. By inhibiting P-gp and probenecid-sensitive transporters, the active and passive processes involved in the distribution and clearance of morphine could be investigated separately $[20,21,25,29,36]$. We find that the concentration profiles of morphine in brain tissue and of its metabolite M3G in plasma are affected by time of day. We show that the transport of morphine from brain tissue back into the circulation shows a characteristic 24-hour rhythm with the lowest efflux during the light-dark phase transitions. The active processes involved in the clearance of morphine and M3G from plasma also show 24-hour variation with the peak in the middle of the dark phase. These findings indicate that dosing time should be taken into account in the optimization of morphine's dosing regimen.

Our results show that inhibition of active transport processes by probenecid and tariquidar alters both the systemic pharmacokinetics and the brain distribution of morphine. With regard to systemic 
pharmacokinetics, inhibition of active transport reduced the systemic clearance of morphine, increased its intercompartmental clearance and lowered the maximal conversion rate of morphine to M3G. Because it was previously found that P-gp inhibition does not influence morphine concentrations in plasma $[21,25,29,37]$, while probenecid treatment has been reported to reduce systemic morphine clearance and the formation of M3G in rats [20], we hypothesize that the systemic effects we observed are due inhibition of multiple multidrug resistance proteins (mrps) in the kidney and liver [38-41] .

Active transport inhibition also altered the brain distribution of morphine. Brain concentrations could be best described by a model in which a "deep brain" compartment was linked to the central plasma compartment by an extra-cellular fluid (ECF) compartment. An additional transport component that was absent inhibitor-treated animals was identified on the transport of morphine from the ECF compartment to plasma. This supports previous findings that morphine is subject to active efflux transport mediated by P-gp and probenecid-sensitive transporters $[20,21,25,29,36]$.

Our findings indicate that several processes involved in morphine pharmacokinetics show 24 -hour variation. It was previously shown in cancer patients that the maximal concentration $\left(\mathrm{C}_{\max }\right)$ and the area under concentration-time profile (AUC) at steady state are higher at 18:00 than at 10:00 and 14:00 after oral administration [15]. In the present study, we have been able to quantify the relative contribution of the processes involved in the distribution, metabolism and elimination of morphine more precisely through the use of six dosing times and the development of a population pharmacokinetic model. We find that the active component of the systemic clearance of morphine and M3G show 24hour variation with a difference of $54 \%$ between the lowest value and the highest value. A physiological explanation of these findings could be the observation that the expression of various probenecid-sensitive transporters show 24-hour variation in the kidney [42]. However, future research to elucidate the underlying mechanisms is warranted.

Furthermore, we find that the transport of morphine from the brain to the blood shows a 12-hour rhythm with the lowest values at the transitions of the light/dark phase. This rhythm could be described by a 24 -hour and 12-hour sinusoidal function on this parameter with a difference between the highest and lowest efflux of $20 \%$. Importantly, the inclusion of this function in the model resolved a time-of-day 
dependent bias observed in the conditionally weighted residuals. In a previous study we found that the efflux of the P-gp substrate quinidine from the brain to plasma is more than two-fold higher during the dark phase compared to the light phase in the presence of functional $\mathrm{P}$-gp transport, but not when $\mathrm{P}$ gp transport is blocked [23]. In the present study, we do not find this P-gp dependent effect for morphine. While quinidine is a selective P-gp substrate, morphine has more complex transport mechanisms across the BBB, which is not only affected by P-gp but also by probenecid-sensitive transporters. The daily variation in P-gp activity may be (partly) counterbalanced by a differentiallyphased variation in probenecid-sensitive transporters. Hence, multiple mechanisms likely give rise to the 12-hour rhythm in the transport of morphine from the brain to blood.

We performed simulations of a single intravenous dose and of a continuous infusion regimen to visualize the effect of the daily rhythmicity in morphine pharmacokinetics on the concentration-time profiles in plasma and brain tissue. Although morphine concentrations in plasma are minimally affected by dosing time, metabolite concentrations in plasma and morphine concentrations in brain tissue do depend on the time of day. This finding has several important implications: it indicates that time of day can be a substantial source of variation in the pharmacokinetics and, possibly, the pharmacodynamics of morphine when it is not properly accounted for, but also that these systematic variations could be exploited to optimize morphine's dosing regimen.

\section{Conflict of interest}

The authors declare no conflicts of interest

\section{Acknowledgments}

The authors would like to thank Ming Liu for her help with the analysis of the samples. This research was supported by the Dutch Technology Foundation STW, which is the applied science division of NWO, and the Technology Programme of the Ministry of Economic Affairs (project number: 12190). The funding source had no involvement in the study design; in the collection, analysis and interpretation of data; in the writing of the report; and in the decision to submit the article for publication. 


\section{References}

[1] E. Sverrisdóttir, T.M. Lund, A.E. Olesen, A.M. Drewes, L.L. Christrup, M. Kreilgaard, A review of morphine and morphine-6-glucuronide's pharmacokinetic-pharmacodynamic relationships in experimental and clinical pain., Eur. J. Pharm. Sci. 74 (2015) 45-62. doi:10.1016/j.ejps.2015.03.020.

[2] R. Dallmann, S.A. Brown, F. Gachon, Chronopharmacology: new insights and therapeutic implications., Annu. Rev. Pharmacol. Toxicol. 54 (2014) 339-61. doi:10.1146/annurevpharmtox-011613-135923.

[3] A.L. Potts, J.F. Cheeseman, G.R. Warman, Circadian rhythms and their development in children: implications for pharmacokinetics and pharmacodynamics in anesthesia., Paediatr. Anaesth. 21 (2011) 238-46. doi:10.1111/j.1460-9592.2010.03343.x.

[4] U. Junker, S. Wirz, Review article: chronobiology: influence of circadian rhythms on the therapy of severe pain., J. Oncol. Pharm. Pract. 16 (2010) 81-7. doi:10.1177/1078155209337665.

[5] R.L. Bornschein, R.S. Crockett, R.P. Smith, Diurnal variations in the analgesic effectiveness of morphine in mice, Pharmacol. Biochem. Behav. 6 (1977) 621-626. doi:10.1016/00913057(77)90085-5.

[6] Y. Cui, K. Sugimoto, N. Araki, T. Sudoh, A. Fujimura, Chronopharmacology of morphine in mice., Chronobiol. Int. 22 (2005) 515-22. doi:10.1081/CBI-200062397.

[7] E.F. Lutsch, R.W. Morris, Light reversal of a morphine-induced analgesia susceptibility rhythm in mice, Experientia. 27 (1971) 420-421. doi:10.1007/BF02137286.

[8] R.W. Morris, E.F. Lutsch, Susceptibility to Morphine-induced Analgesia in Mice, Nature. 216 (1967) 494-495. doi:10.1038/216494a0.

[9] M. Yoshida, S. Ohdo, H. Takane, Y. Tomiyoshi, A. Matsuo, E. Yukawa, S. Higuchi, Chronopharmacology of analgesic effect and its tolerance induced by morphine in mice., J. Pharmacol. Exp. Ther. 305 (2003) 1200-5. doi:10.1124/jpet.103.049031.

[10] M. Yoshida, S. Koyanagi, A. Matsuo, T. Fujioka, H. To, S. Higuchi, S. Ohdo, Glucocorticoid hormone regulates the circadian coordination of micro-opioid receptor expression in mouse brainstem., J. Pharmacol. Exp. Ther. 315 (2005) 1119-24. doi:10.1124/jpet.105.091488. 
[11] M. Kavaliers, M. Hirst, Daily rhythms of analgesia in mice: effects of age and photoperiod, Brain Res. 279 (1983) 387-393. doi:10.1016/0006-8993(83)90216-0.

[12] A. Oliverio, C. Castellano, S. Puglisi-Allegra, Opiate analgesia: Evidence for circadian rhythms in mice, Brain Res. 249 (1982) 265-270. doi:10.1016/0006-8993(82)90060-9.

[13] S. Dohoo, Steady-state pharmacokinetics of oral sustained-release morphine sulphate in dogs, J. Vet. Pharmacol. Ther. 20 (1997) 129-133. doi:10.1046/j.1365-2885.1997.00816.x.

[14] N.A. Rasmussen, L.A. Farr, Effects of Morphine and Time of Day on Pain and Beta-Endorphin, Biol. Res. Nurs. 5 (2003) 105-116. doi:10.1177/1099800403257166.

[15] G.K. Gourlay, J.L. Plummer, D.A. Cherry, Chronopharmacokinetic variability in plasma morphine concentrations following oral doses of morphine solution, Pain. 61 (1995) 375-381. doi:10.1016/0304-3959(94)00204-R.

[16] S. Dohoo, Steady-state pharmacokinetics of oral sustained-release morphine sulphate in dogs, J. Vet. Pharmacol. Ther. 20 (1997) 129-133. doi:10.1046/j.1365-2885.1997.00816.x.

[17] S. De Gregori, M. De Gregori, G.N. Ranzani, M. Allegri, C. Minella, M. Regazzi, Morphine metabolism, transport and brain disposition, Metab. Brain Dis. 27 (2011) 1-5. doi:10.1007/s11011-011-9274-6.

[18] A. a Somogyi, D.T. Barratt, J.K. Coller, Pharmacogenetics of opioids., Clin. Pharmacol. Ther. 81 (2007) 429-44. doi:10.1038/sj.clpt.6100095.

[19] S.J. Thompson, K. Koszdin, C.M. Bernards, Opiate-induced Analgesia Is Increased and Prolonged in Mice Lacking P-glycoprotein, Anesthesiology. 92 (2000) 1392-1399. doi:10.1097/00000542-200005000-00030.

[20] K. Tunblad, E.N. Jonsson, M. Hammarlund-Udenaes, Morphine Blood-Brain Barrier Transport Is Influenced by Probenecid Co-Administration, Pharm. Res. 20 (2003) 618-623. doi:10.1023/A:1023250900462.

[21] R. Xie, M. Hammarlund-Udenaes, A.G. de Boer, E.C. de Lange, The role of P-glycoprotein in blood-brain barrier transport of morphine: transcortical microdialysis studies in mdr1a (-/-) and mdr1a (+/+) mice., Br. J. Pharmacol. 128 (1999) 563-8. doi:10.1038/sj.bjp.0702804.

[22] Y.-K.J. Zhang, R.L. Yeager, C.D. Klaassen, Circadian expression profiles of drug-processing 
genes and transcription factors in mouse liver., Drug Metab. Dispos. 37 (2009) 106-15. doi:10.1124/dmd.108.024174.

[23] L. Kervezee, R. Hartman, D.-J. van den Berg, S. Shimizu, Y. Emoto-Yamamoto, J.H. Meijer, E.C.M. de Lange, Diurnal Variation in P-glycoprotein-Mediated Transport and Cerebrospinal Fluid Turnover in the Brain, AAPS J. 16 (2014) 1029-1037. doi:10.1208/s12248-014-9625-4.

[24] J. Westerhout, B. Ploeger, J. Smeets, M. Danhof, E.C.M. de Lange, Physiologically based pharmacokinetic modeling to investigate regional brain distribution kinetics in rats., AAPS J. 14 (2012) 543-53. doi:10.1208/s12248-012-9366-1.

[25] D. Groenendaal, J. Freijer, D. de Mik, M.R. Bouw, M. Danhof, E.C.M. de Lange, Population pharmacokinetic modelling of non-linear brain distribution of morphine: influence of active saturable influx and P-glycoprotein mediated efflux., Br. J. Pharmacol. 151 (2007) 701-12. doi:10.1038/sj.bjp.0707257.

[26] R.J. Keizer, R.S. Jansen, H. Rosing, B. Thijssen, J.H. Beijnen, J.H.M. Schellens, A.D.R. Huitema, Incorporation of concentration data below the limit of quantification in population pharmacokinetic analyses., Pharmacol. Res. Perspect. 3 (2015) e00131. doi:10.1002/prp2.131.

[27] U. Bickel, O.P. Schumacher, Y.S. Kang, K. Voigt, Poor permeability of morphine 3-glucuronide and morphine 6-glucuronide through the blood-brain barrier in the rat., J. Pharmacol. Exp. Ther. 278 (1996) 107-13. http://www.ncbi.nlm.nih.gov/pubmed/8764341 (accessed December 7, 2015).

[28] E. Boström, M. Hammarlund-Udenaes, U.S.H. Simonsson, Blood-brain barrier transport helps to explain discrepancies in in vivo potency between oxycodone and morphine., Anesthesiology. 108 (2008) 495-505. doi:10.1097/ALN.0b013e318164cf9e.

[29] S.P. Letrent, G.M. Pollack, K.R. Brouwer, K.L.R. Brouwer, Effects of a potent and specific Pglycoprotein inhibitor on the blood-brain barrier distribution and antinociceptive effect of morphine in the rat., Drug Metab. Dispos. 27 (1999) 827-34.

[30] F. Stain-Texier, G. Boschi, P. Sandouk, J.M. Scherrmann, Elevated concentrations of morphine 6-beta-D-glucuronide in brain extracellular fluid despite low blood-brain barrier permeability., Br. J. Pharmacol. 128 (1999) 917-24. doi:10.1038/sj.bjp.0702873. 
[31] K. Tunblad, M. Hammarlund-Udenaes, E.N. Jonsson, An Integrated Model for the Analysis of Pharmacokinetic Data from Microdialysis Experiments, Pharm. Res. 21 (2004) 1698-1707. doi:10.1023/B:PHAM.0000041468.00587.c6.

[32] S. Beal, L.B. Sheiner, A. Boeckmann, R.J. Bauer, NONMEM User's Guides (1989-2009), Icon Development Solutions, Ellicott City, MD, USA, (2009).

[33] R.J. Keizer, M.O. Karlsson, A. Hooker, Modeling and Simulation Workbench for NONMEM: Tutorial on Pirana, PsN, and Xpose., CPT Pharmacometrics Syst. Pharmacol. 2 (2013) e50. doi:10.1038/psp.2013.24.

[34] D.R. Mould, R.N. Upton, Basic concepts in population modeling, simulation, and model-based drug development-part 2: introduction to pharmacokinetic modeling methods., CPT Pharmacometrics Syst. Pharmacol. 2 (2013) e38. doi:10.1038/psp.2013.14.

[35] M.R. Bouw, M. Gårdmark, M. Hammarlund-Udenaes, Pharmacokinetic-Pharmacodynamic Modelling of Morphine Transport Across the Blood-Brain Barrier as a Cause of the Antinociceptive Effect Delay in Rats—A Microdialysis Study, Pharm. Res. 17 (2000) 12201227. doi:10.1023/A:1026414713509.

[36] F. Bourasset, J.-M. Scherrmann, Carrier-mediated processes at several rat brain interfaces determine the neuropharmacokinetics of morphine and morphine-6-beta-D-glucuronide., Life Sci. 78 (2006) 2302-14. doi:10.1016/j.lfs.2005.09.043.

[37] S.P. Letrent, G.M. Pollack, K.R. Brouwer, K.L.R. Brouwer, Effect of GF120918, a Potent Pglycoprotein Inhibitor, on Morphine Pharmacokinetics and Pharmacodynamics in the Rat, Pharm. Res. 15 (1998) 599-605. doi:10.1023/A:1011938112599.

[38] M.J. Dresser, M.K. Leabman, K.M. Giacomini, Transporters involved in the elimination of drugs in the kidney: Organic anion transporters and organic cation transporters, J. Pharm. Sci. 90 (2001) 397-421. doi:10.1002/1520-6017(200104)90:4<397::AID-JPS1000>3.0.CO;2-D.

[39] N. Zelcer, T. Saeki, G. Reid, J.H. Beijnen, P. Borst, Characterization of drug transport by the human multidrug resistance protein 3 (ABCC3)., J. Biol. Chem. 276 (2001) 46400-7. doi:10.1074/jbc.M107041200.

[40] N. Zelcer, K. van de Wetering, M. Hillebrand, E. Sarton, A. Kuil, P.R. Wielinga, T. Tephly, A. 
Dahan, J.H. Beijnen, P. Borst, Mice lacking multidrug resistance protein 3 show altered morphine pharmacokinetics and morphine-6-glucuronide antinociception., Proc. Natl. Acad. Sci. U. S. A. 102 (2005) 7274-9. doi:10.1073/pnas.0502530102.

[41] W. Lee, R.B. Kim, Transporters and renal drug elimination., Annu. Rev. Pharmacol. Toxicol. 44 (2004) 137-66. doi:10.1146/annurev.pharmtox.44.101802.121856.

[42] F. Gachon, D. Firsov, The role of circadian timing system on drug metabolism and detoxification., Expert Opin. Drug Metab. Toxicol. 7 (2011) 147-58. doi:10.1517/17425255.2011.544251. 


\section{Figure legends}

Figure 1 Concentration profiles of morphine (MOR) in plasma (upper panels), M3G in plasma (middle panels) and MOR in brain tissue (lower panels) in vehicle-treated (left) and inhibitor-treated animals (right) after different dosing times.

Figure 2: Structure of the final combined plasma-brain model. Colored compartments indicated the site of sampling (red: morphine concentrations in plasma; green: M3G concentrations in plasma; blue: morphine concentrations in brain tissue).

Figure 3 Visual predictive check (VPC) stratified by treatment group. Dots: observed data; solid line: median of the predicted concentrations; shaded areas enclosed by dashed lines: $90 \%$ prediction intervals of the simulated data.

Figure 4 Distribution of CWRESI vs time of dose in vehicle-treated (dark symbols) and probenecid (PRB) - tariquidar (TQD) treated (light symbols) animals. A, C, E: CWRESI distribution in the model without cosine functions of morphine concentrations in brain $(A)$ and in plasma $(C)$ and of M3G concentrations in plasma (E). B: CWRESI distribution in the model with a 24+12-hour cosine included on $Q_{E C F-P L}$ of morphine concentrations in brain. D and F: CWRESI distribution in the model with a 24+12-hour cosine function included on $\mathrm{CL}_{10, \text { active }}$ and $\mathrm{CL}_{\mathrm{M} 3 \mathrm{G} \text {, active }}$ of morphine concentrations in plasma (D) and of M3G concentrations in plasma $(F)$.

Figure 5 Shape of the cosine functions included on $\mathrm{Q}_{\mathrm{ECF}-\mathrm{PL}}$ (left), $\mathrm{CL}_{10 \text {,active }}$ (middle) and $\mathrm{CL}_{\mathrm{M} 3 \mathrm{G} \text {,active }}$ (right).

Figure 6 Measured versus population predicted (PRED; upper panels) and individual predicted (IPRED; lower panels) concentrations of morphine (MOR) in plasma (left, red), M3G in plasma (middle, green) and MOR in brain tissue (right, blue) of the final model. Dark coloured symbols represent vehicle-treated animals; light coloured symbols represent inhibitor-treated animals. Dotted line: line of unity. 
Figure 7 Simulations of morphine (MOR) concentration-time profiles in plasma (left), M3G concentrations in plasma (middle) and morphine concentrations in brain (right) after a 10 min. intravenous infusion of $4 \mathrm{mg} / \mathrm{kg}$ at six different dosing times (start of infusion at $\mathrm{t}=0,4,8,12,16,20$ hours after light onset) (a) and during a continuous infusion of $1 \mathrm{mg} / \mathrm{kg} / \mathrm{h}$ started at 0 hours after light onset (b). 


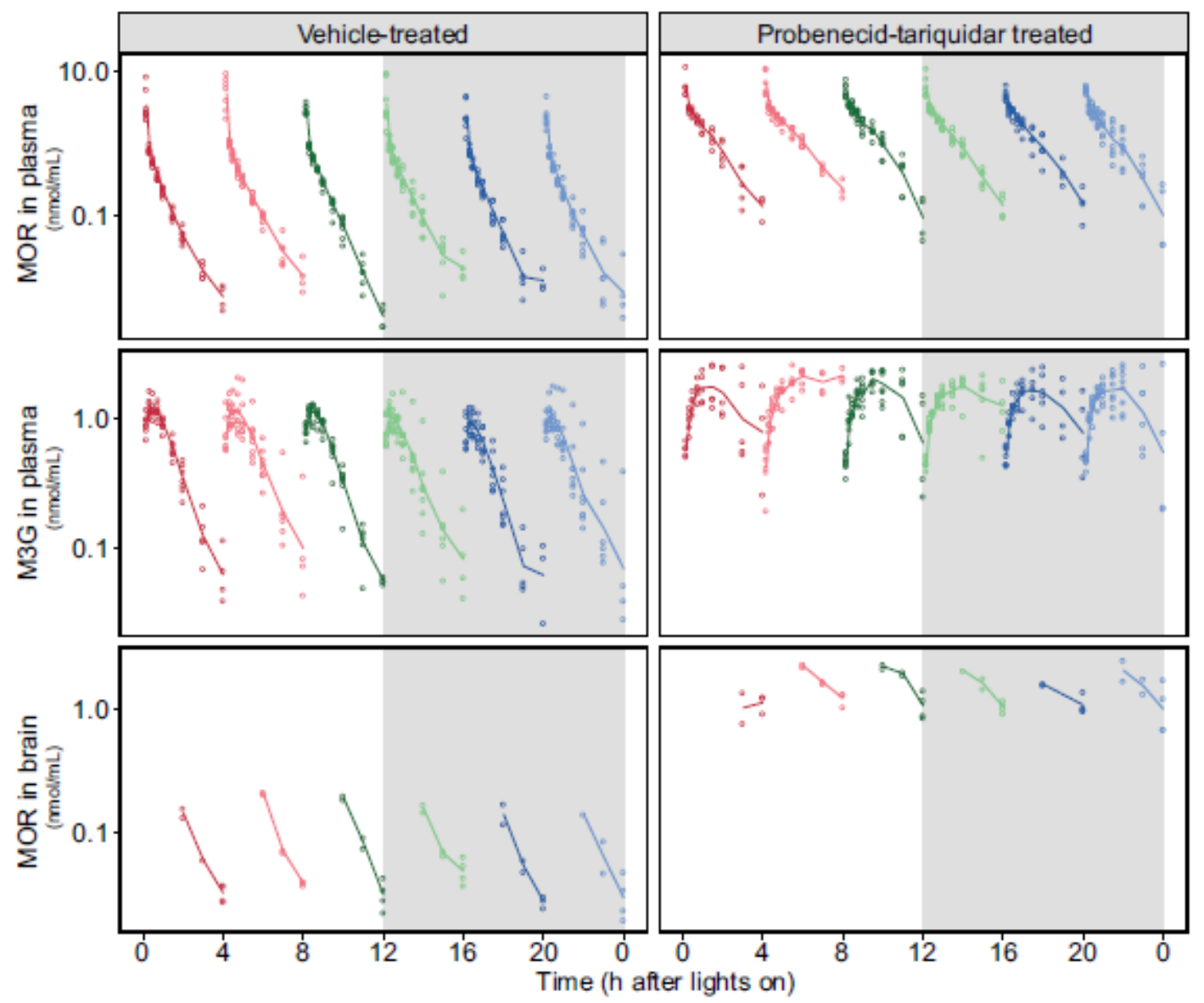

Figure 1 


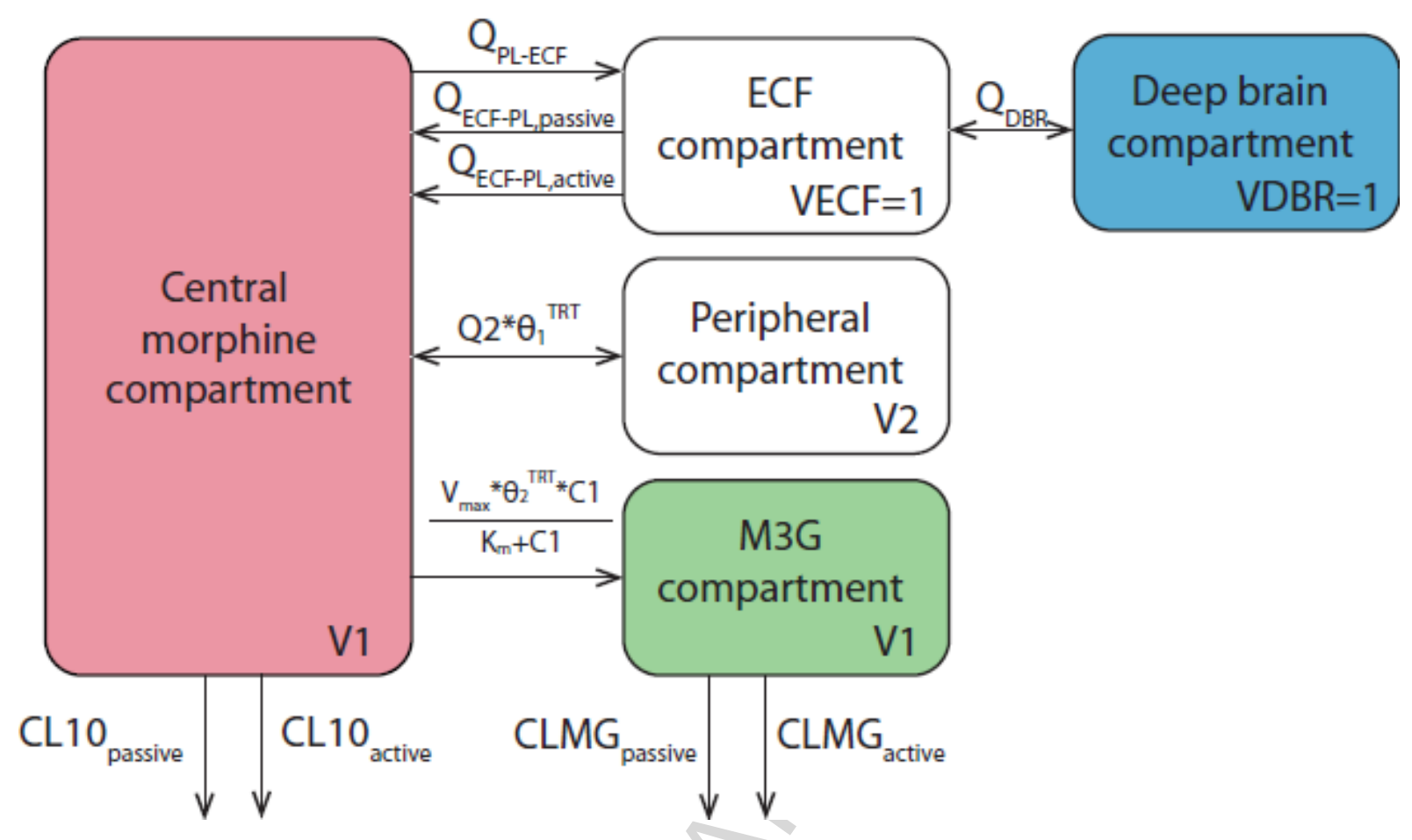

Figure 2 


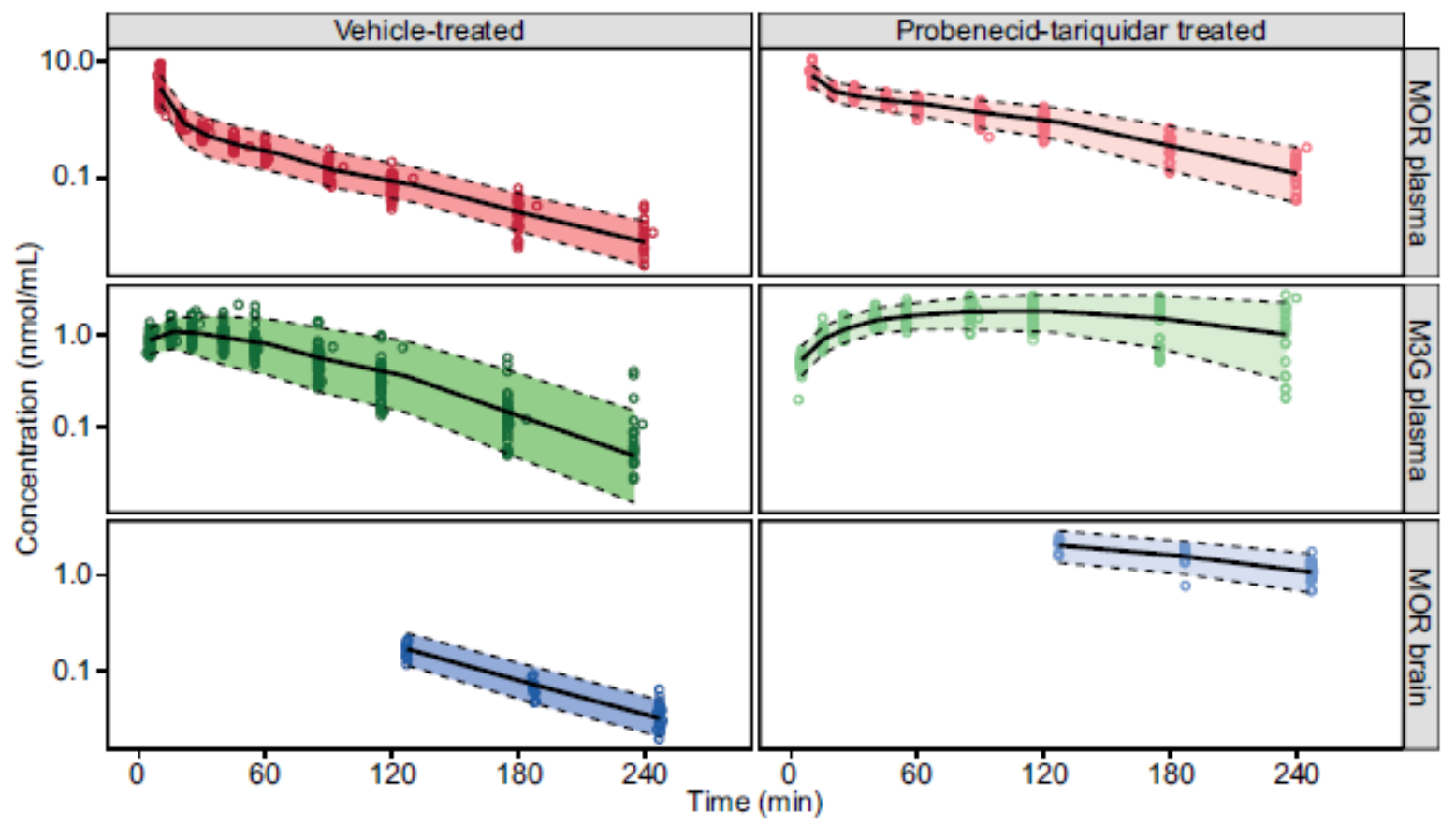

Figure 3 

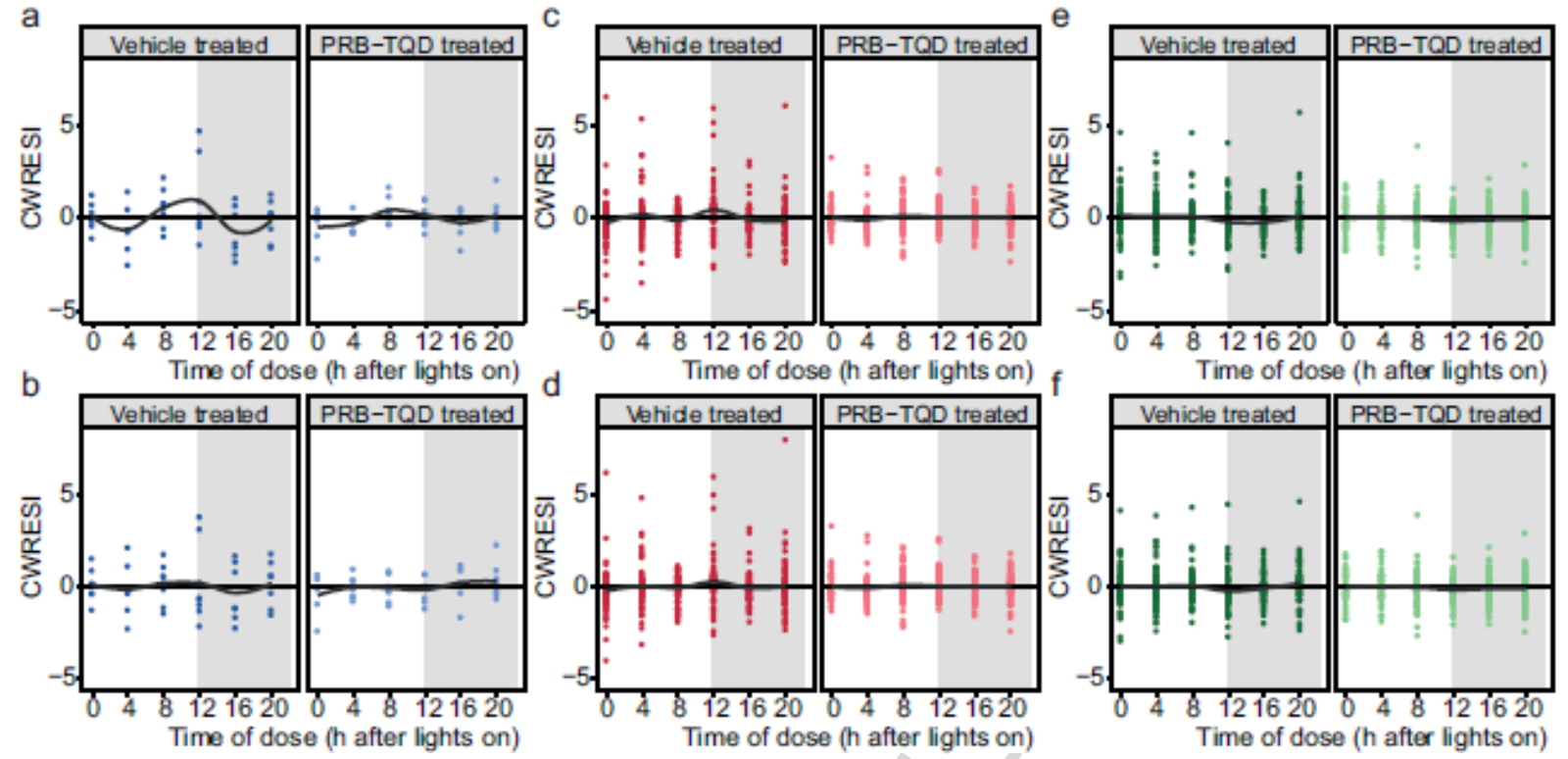

Figure 4 

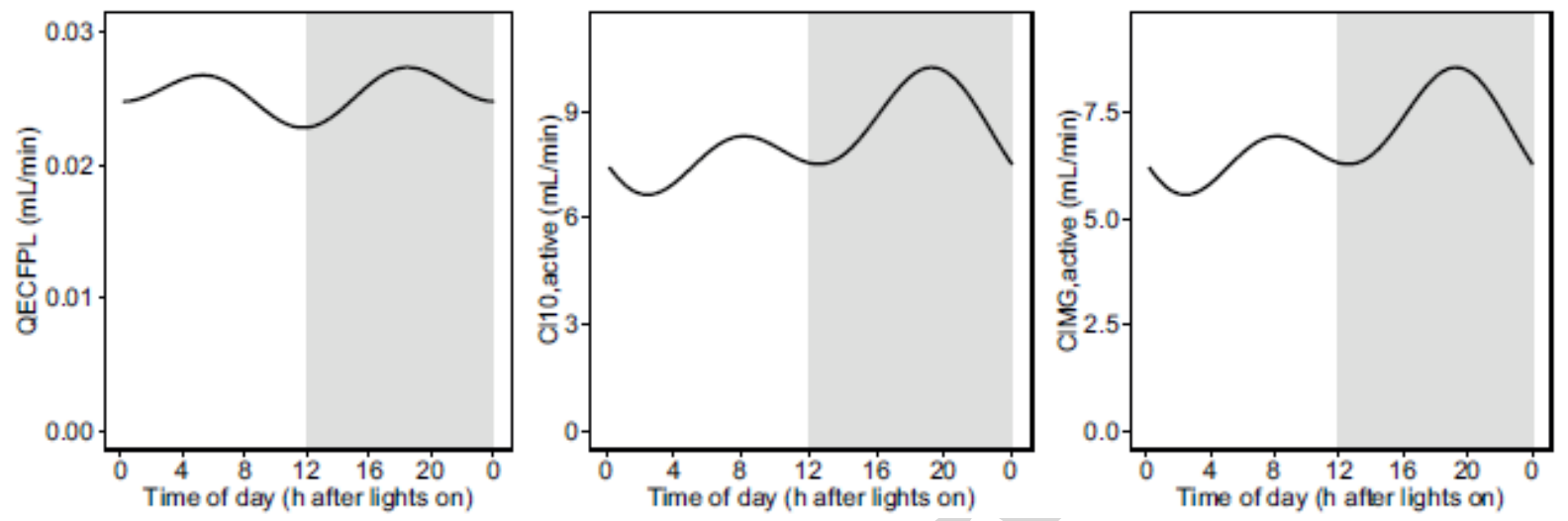

Figure 5 

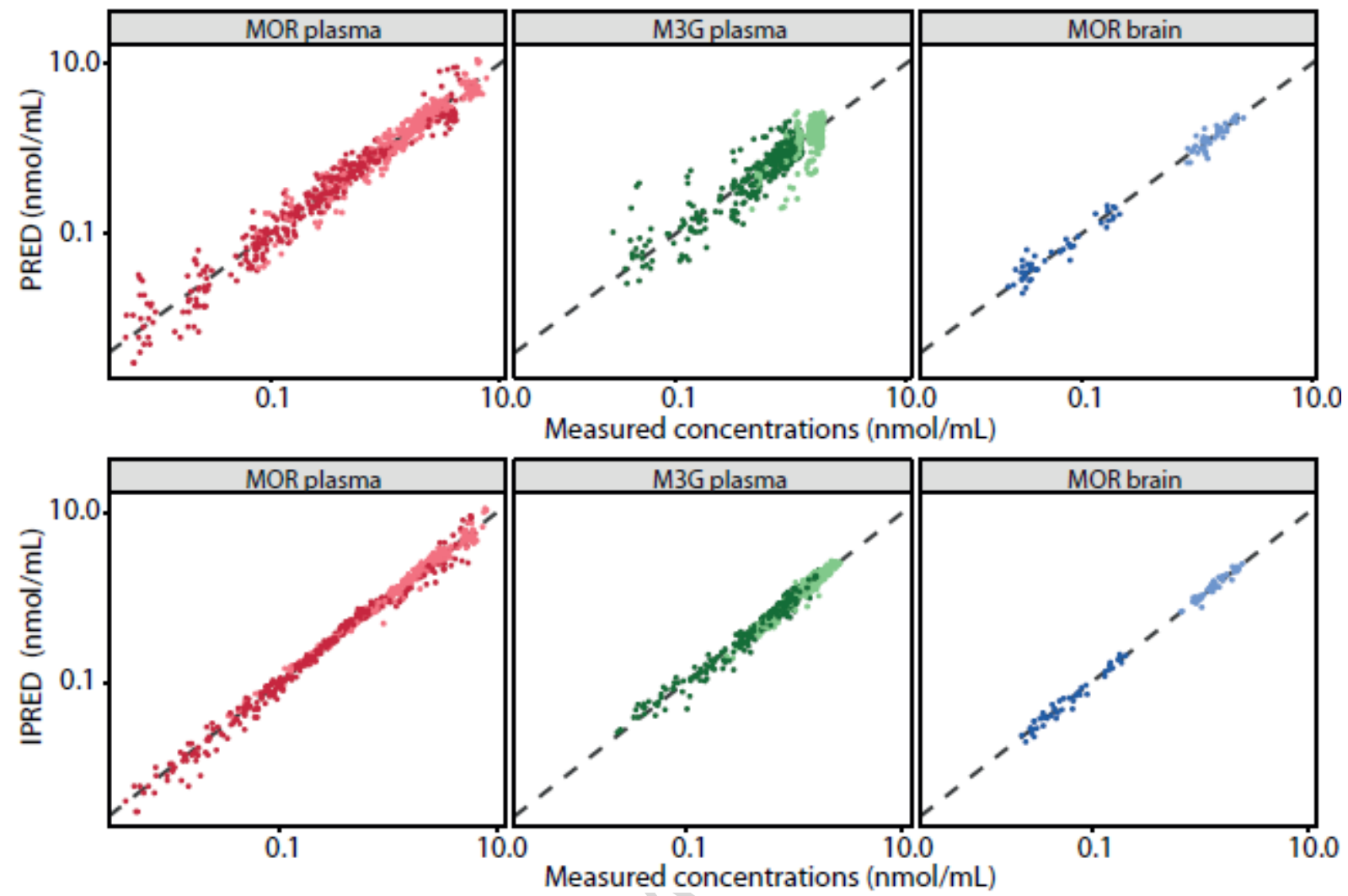

Figure 6 

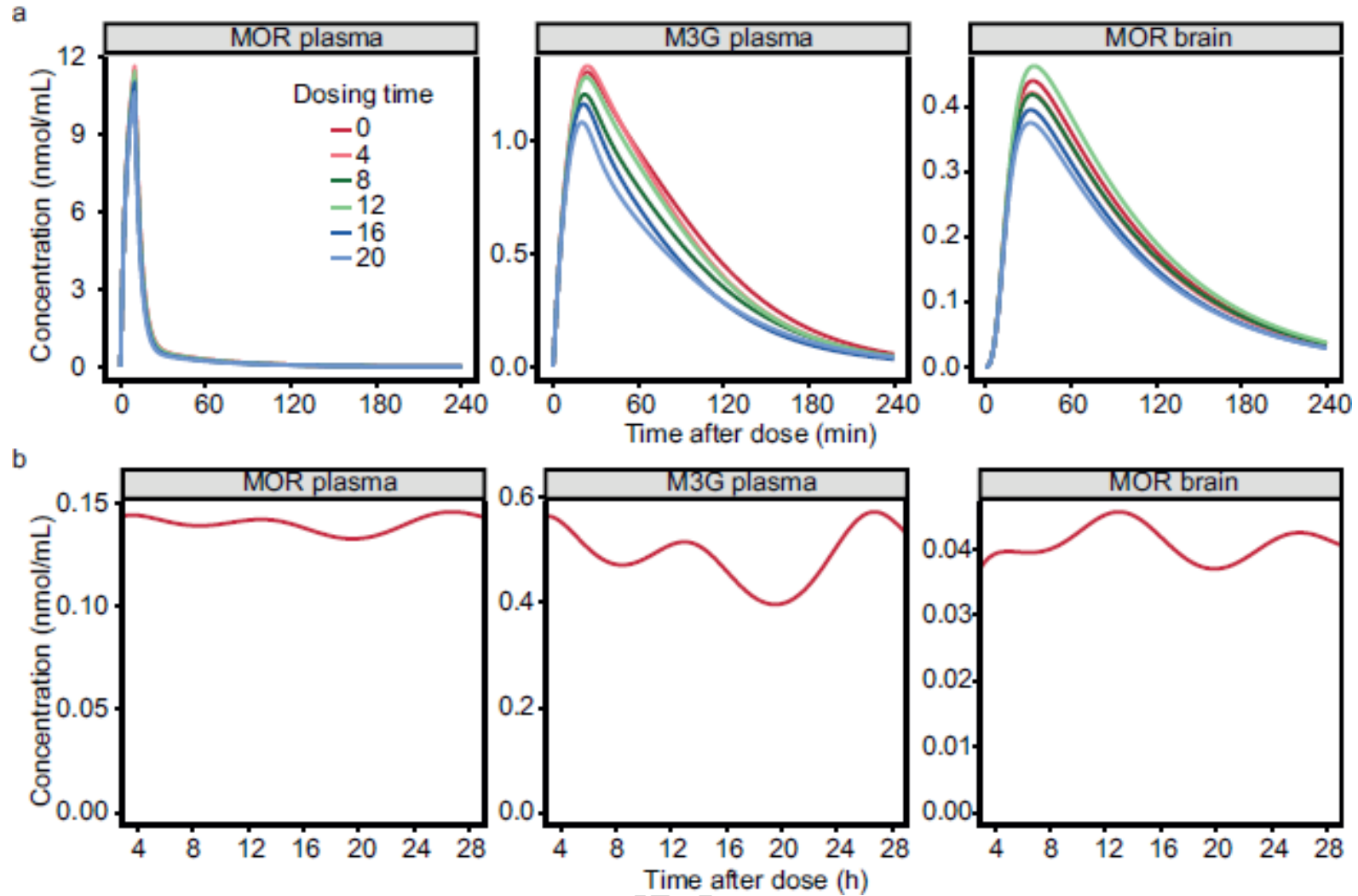

Figure 7 
Table 1 Number of animals per treatment group

\begin{tabular}{|c|c|c|}
\hline Dosing time & No. of vehicle-treated animals & No. of inhibitor-treated animals \\
\hline 0 & 7 & 5 \\
\hline 4 & 8 & 7 \\
\hline 8 & 8 & 8 \\
\hline 12 & 8 & 7 \\
\hline 16 & 8 & 7 \\
\hline 20 & 8 & 8 \\
\hline TOTAL & 47 & 42 \\
\hline
\end{tabular}


Table 2 Parameter estimates of combined plasma and brain model (with 24-hour variation included on $\mathrm{CL}_{10 \text {,active, }} \mathrm{CL}_{\mathrm{M} 3 \mathrm{G} \text {,active }}$ and $\left.\mathrm{Q}_{\mathrm{ECF}-\mathrm{PL}}\right)$ and results from bootstrap analysis (444/500 resamples successful)

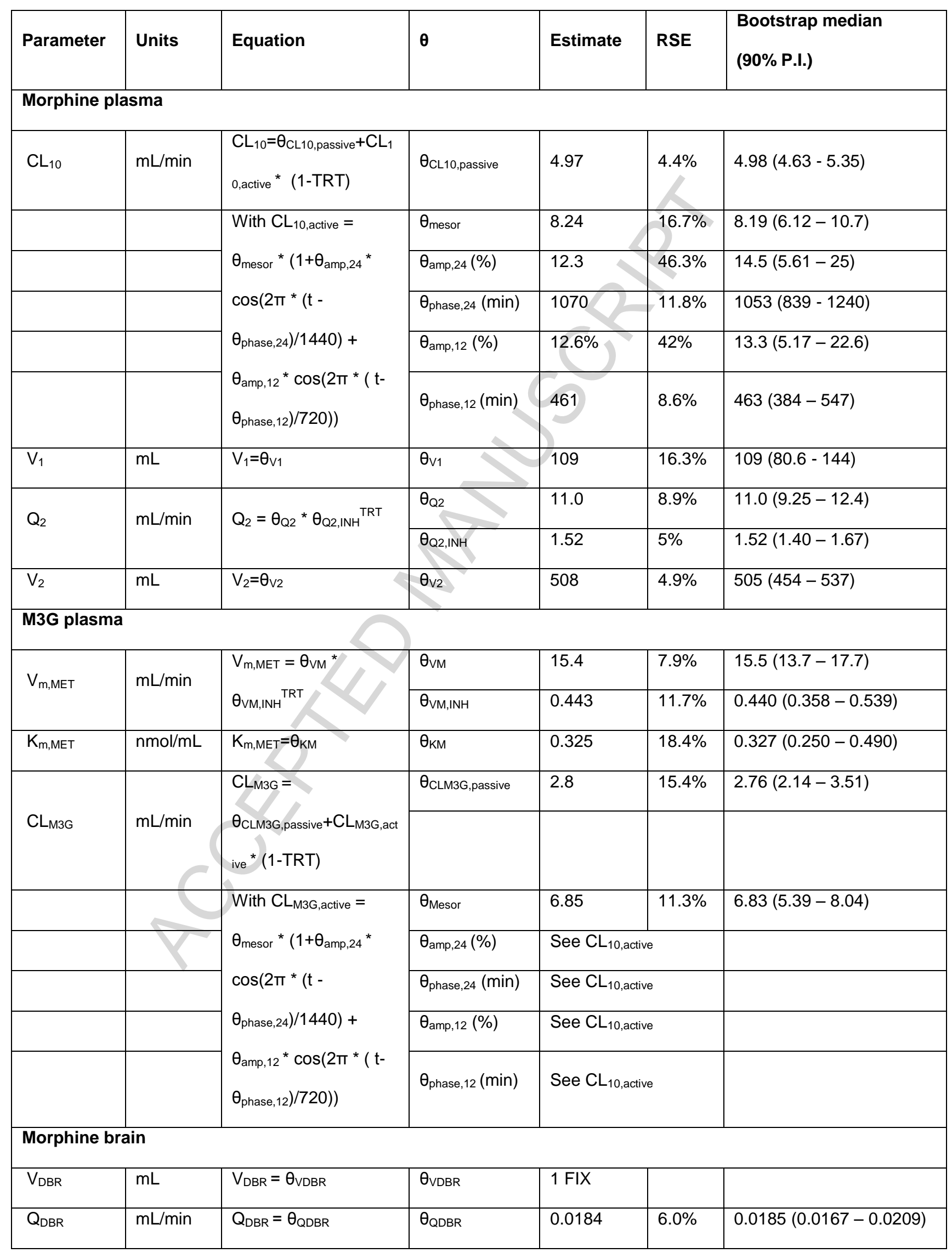




\begin{tabular}{|c|c|c|c|c|c|c|}
\hline $\mathrm{V}_{\mathrm{ECF}}$ & $\mathrm{mL}$ & $\mathrm{V}_{\mathrm{ECF}}=\theta_{\mathrm{VECF}}$ & $\theta_{\text {VECF }}$ & $1 \mathrm{FIX}$ & & \\
\hline \multirow{6}{*}{ QECF-PL } & \multirow{2}{*}{$\mathrm{mL} / \mathrm{min}$} & \multirow{3}{*}{$\begin{array}{l}Q_{E C F-P L}= \\
\left(\theta_{\text {QECFPL,passive }}+\right. \\
\left.Q_{E C F P L, a c t i v e}^{*}(1-T R T)\right)\end{array}$} & $\theta_{\mathrm{QECFPL}, \text { passive }}$ & 0.0256 & $9.5 \%$ & $0.0251(0.0211-0.0301)$ \\
\hline & & & $\theta_{\text {QECFPL,active }}$ & 0.0834 & $12.4 \%$ & $0.0824(0.0659-0.103)$ \\
\hline & & & $\theta_{\mathrm{amp}, 24}(\%)$ & $3.76 \%$ & $42 \%$ & $4.33(1.75-6.82)$ \\
\hline & & ${ }^{*}\left(1+\theta_{\mathrm{amp}, 24}{ }^{*} \cos \left(2 \pi{ }^{*}\right.\right.$ & $\theta_{\text {phase }, 24}(\min )$ & 1390 & $7.4 \%$ & $1390(1150-1600)$ \\
\hline & & $\left.\left(\mathrm{t}-\theta_{\text {phase,24 }}\right) / 1440\right)+$ & $\theta_{a m p, 12(\%)}$ & $6.33 \%$ & $32.2 \%$ & $6.76(3.79-10.5)$ \\
\hline & & $\begin{array}{l}\theta_{\text {amp }, 12} \cos (2 \pi \\
\left.\left.\left.\theta_{\text {phase, } 12}\right) / 720\right)\right)\end{array}$ & $\theta_{\text {phase }, 12}(\min )$ & 1060 & $3.3 \%$ & $1060(990-1120)$ \\
\hline QPL-ECF & $\mathrm{mL} / \mathrm{min}$ & $Q_{P L-E C F}=\theta_{\text {QPLECF }}$ & $\theta_{\mathrm{QPLECF}}$ & 0.0322 & $10.5 \%$ & $0.0316(0.260-0.0386)$ \\
\hline Inter-anim & ariabilit & V\%) & & & & \\
\hline$\omega^{2} \mathrm{CL}_{10}$ & & & & 17.3 & & 16.8 \\
\hline$\omega^{2} V_{m, M E T}$ & & & & 22.2 & & 21.9 \\
\hline$\omega^{2} C_{M G}$ & & & & 43.5 & & 42.6 \\
\hline$\omega^{2} \mathrm{Q} 2$ & & & & 18.7 & & 18.4 \\
\hline$\omega^{2} V_{m, M E T}$ & ${ }^{2} \mathrm{CL}_{\mathrm{MG}}(\mathrm{u}$ & ansformed) & & 0.0761 & & 0.0742 \\
\hline Residual & xplained & riability (\%) & & & & \\
\hline$\sigma_{\mathrm{PL}}$ & & & & 17.0 & & 16.8 \\
\hline$\sigma_{\mathrm{M} 3 \mathrm{G}}$ & & & & 14.5 & & 14.4 \\
\hline$\sigma_{\mathrm{DBR}}$ & & & & 11.8 & & 11.3 \\
\hline
\end{tabular}


Graphical abstract

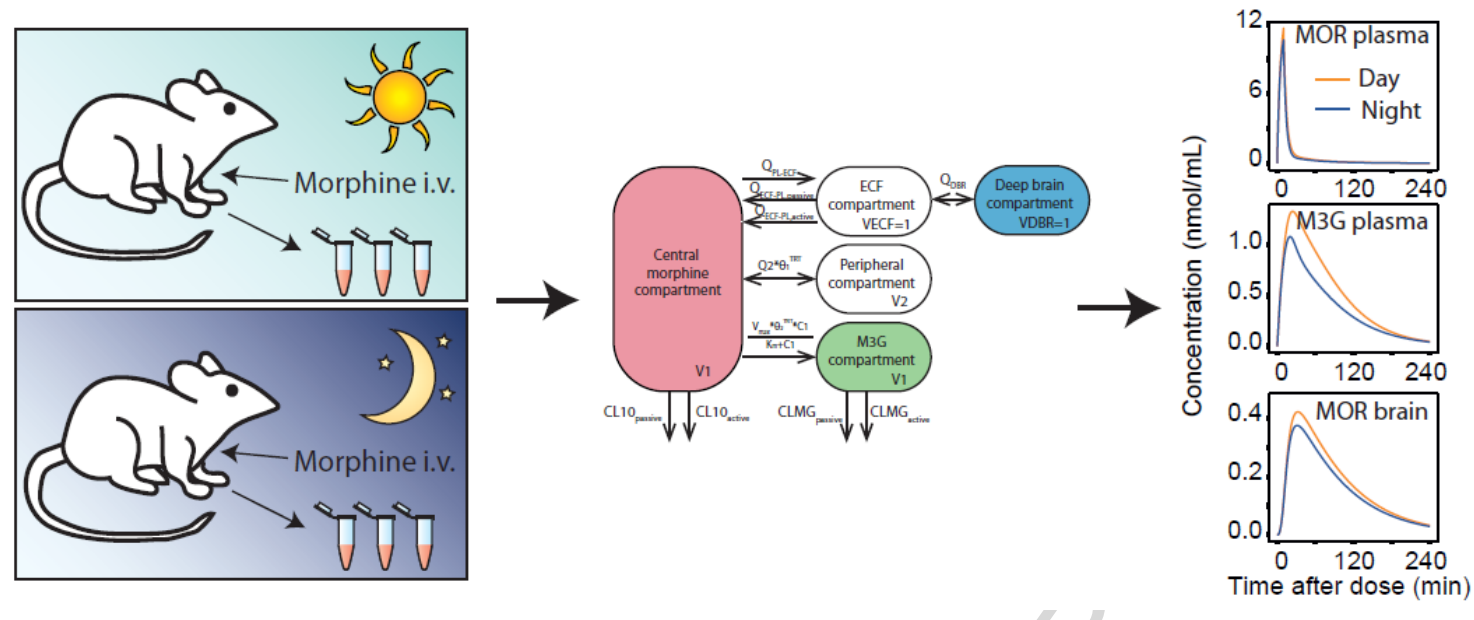

